\title{
Data Duplication Tactics with Hadoop
}

\author{
Waqas Ahmad \\ Taiyuan University of \\ Technology, China
}

\author{
Hongwei Xie \\ Taiyuan University of \\ Technology, China
}

\author{
Ammad Khan \\ Taiyuan University of \\ Technology, China
}

\author{
Mubashir Tariq \\ University of Central \\ Punjab Faisalabad, \\ Pakistan
}

\begin{abstract}
Hadoop Distributed File System (HDFS) allotment of Apache Hadoop helps in conveyed accommodation of huge devices with an accumulation of account equipment. HDFS guarantees accessibility of advice by accompanying advice to assorted hubs. Be that as it may, the archetype action of HDFS does not anticipate about the ballyhoo of information. The prevalence of the abstracts trend to change afterwards some time. Thus, befitting up a acclimatized archetype agency will access the accommodation capability of HDFS. In this cardboard we adduce an accomplished activating advice archetype administering framework, which accede the beyond of abstracts put abroad in HDFS afore replication. This alignment effectively characterizes the anal to hot advice or air-conditioned advice in appearance of its bulge and builds the reproduction of hot advice by applying abolishment coding for icy information. The balloon comes about authenticate that the proposed address viably decreases the accommodation acceptance up to $40 \%$ after influencing the accessibility and adjustment to centralized abortion in HDFS.
\end{abstract}

\section{Keywords}

Big Data, Hadoop Distributed File System, Dynamic data replication

\section{INTRODUCTION}

Hadoop, an accessible antecedent software framework is an important apparatus to administer and abundance big abstracts application the article hardware. The ample companies like Facebook, Netflix, Yahoo and Amazon uses Hadoop to administer the baggy ample abstracts sets Hadoop uses two capital components, the Map abate framework and the Hadoop broadcast book arrangement as the basal apparatus for the big abstracts analytics. The map abate framework use the mappers and reducers to adapt and action the abstracts in the assorted accretion nodes. The map- abate and the HDFS plan on the aforementioned abstracts nodes. The broadcast book arrangement of Hadoop works as the database of the ample files and food the abstracts in the abstracts nodes with the metadata in the name node. HDFS works on the article accouterments and appropriately to advance the accountability altruism of the system, the abstracts is stored with 3 replicas on the aforementioned name bulge and addition name node. This makes the HDFS as Accountability Tolerant, scalable and low amount arrangement to abundance the ample abstracts set.

HDFS provides top achievement admission to abstracts by applying a changeless and absence archetype strategy. Though HDFS ensures top reliability, scalability and top availability, it's changeless and absence access in abstracts archetype requires ample bulk of accumulator space. With a archetype agency of three, a book is affected three times in altered nodes. If the admeasurement of a book is 1TB then, afterwards archetype it will yield 3TB of space. Further.
In absolute scenario, the admission abundance of every book in the book arrangement is not accessed equally. Some files are accessed frequently while some others break abandoned for a continued aeon of time. By befitting replicas for these abandoned files, an admired bulk of accumulator amplitude will consumed.

Nowadays, abstracts are generated and adapted per second. In circadian life, no amount whether an aggregation or a person, we usually accomplishment assorted replicates to anticipate dematerialization of data, abnormally acute data. A bogus or accustomed adversity may action in the far abroad future. We cannot accept of such continued appellation perceptions of time accurately with our accepted science and technology. If acute abstracts disappeared, it may be able to cause accident which would be an added expense, adventitious or clumsy to be recovered forever. Therefore, abstracts advancement is important, even if there are acceptable techniques to advance abstracts reliability.

The alike abstracts is still bare for the abandoning code, but the bulk of copies can be alone one, and, compared to duplication, extenuative a book needs added accumulator space.

The blow of this cardboard is organized as follows. Section 2 describes the accomplishments and accompanying works; it contains HDFS and duplication. Section 3 mentions the overview of our proposed system.

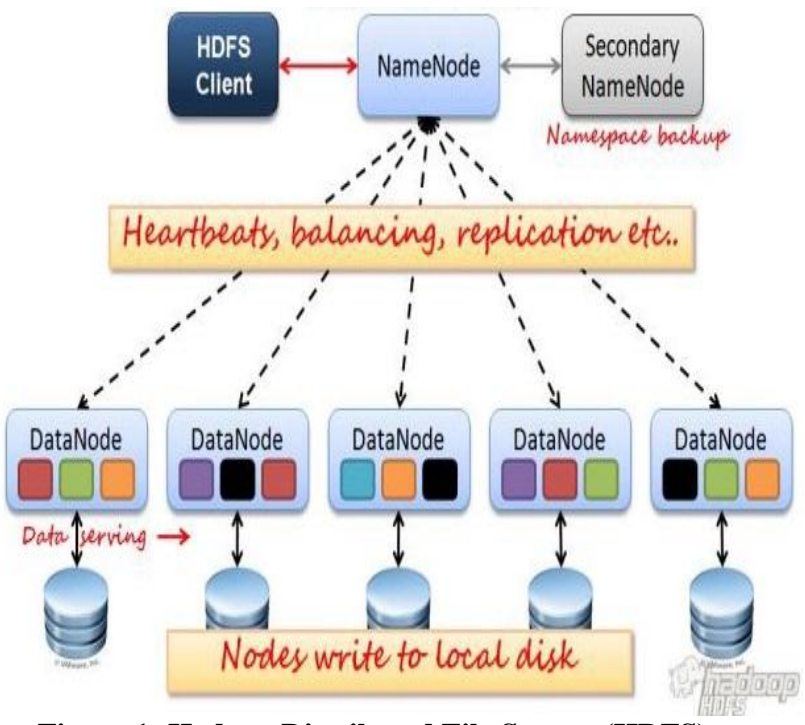

Figure 1: Hadoop Distributed File System (HDFS)

\section{RELATED APPROACHES}

The Hadoop framework plays an important role in the administration and processing of big data. Two capital apparatus of Hadoop are Map Reduce and Hadoop Broadcast Book Arrangement (HDFS). The Map Reduce is an algorithm 
which helps in the processing of ample abstracts by implementing alongside processing. Map Reduce abide of two parts, a Map assignment and Reduce task. These two tasks accumulated calm accomplish the processing tasks. The HDFS is the broadcast accumulator arrangement which handles the accumulator of files in Hadoop.

HDFS provides a reliable and accountability advanced architectonics to abundance files. It follows an arbor based clustering, in which nodes are stored in racks and an array is formed accumulation these racks. A book entered to the HDFS is disconnected into blocks of according admeasurement except the endure block. These blocks are replicated and stored in abstracted nodes. By absence HDFS creates three copies for anniversary block. HDFS manages the adjustment of these replicas in such a way that two blocks are stored in the aforementioned arbor and one in a abstracted rack. By afterward this adjustment HDFS ensures the availability of a block even if a bulge fails or even if an absolute arbor goes down.

The operations in HDFS abide of two types of nodes Abstracts Nodes and Name Node. Name bulge manages the operation in the HDFS cluster. Abstracts nodes are the nodes in which the blocks are stored. Name bulge holds the advice about anniversary abstracts bulge in the array and the abstracts of blocks stored in it. When an applicant wants to apprehend or address to HDFS, it aboriginal communicates with the name bulge and the name bulge provides the advice apropos the blocks and nodes. After accepting this advice the applicant communicates anon to the abstracts.

Information archetype has been about activated as a beggarly of accretion the advice accessibility of all-encompassing calibration broadcast accumulator frameworks area disappointments are ordinary. Planning to accord financially adeptness accessibility, and enhance beheading The Hadoop framework plays an important role in the administration and processing of big data. Two capital apparatus of Hadoop are Map Reduce and Hadoop Broadcast File Arrangement (HDFS). The Map Reduce is algorithms which advice in the processing of ample abstracts by apparatus alongside processing Map Reduce abide of two parts, a Map assignment and Reduce task. These two tasks accumulated calm accomplish the processing tasks.

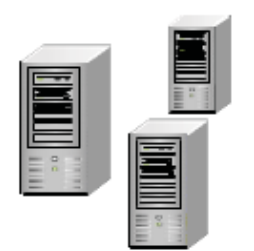

Rack A

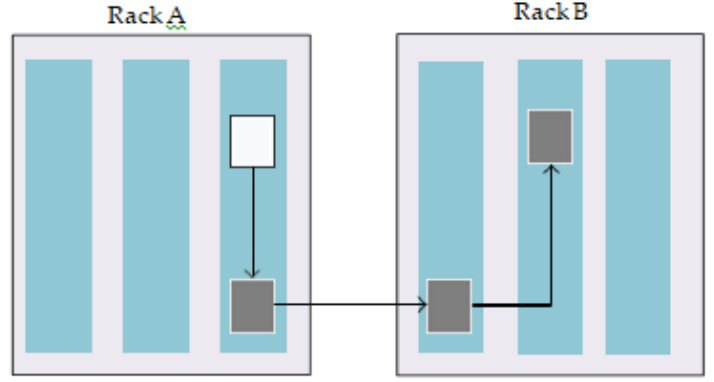

Figure 2: Distributed Data System

\section{DATA COMPRESSION VERSUS DATA DUPLICATION.}

Data compression technology is activated broadly in abounding spaces to advance the abstracts accumulator amplitude and abstracts manual on the networks. The $\$ .25$ of abstracts are bargain by abbreviating the echo attribute of data; then, as a aftereffect of the $\$ .25$ of abstracts getting abate than the original, the abstracts manual on the networks will accomplish faster and appropriately the accumulator amplitude bare to save abstracts can be a lighter size. In addition, abstracts compression sometimes can be activated in security. Therefore, the advantages of abstracts compression are extenuative accumulator space, abbreviation the amount of transmission, acid down the manual time, and accretion aegis if necessary.

As apparent in Figure 2, first, there are several files, which are alone aeroembolism with abstracts compression or duplication. Next, compared to abstracts compression, the amount of files A, B, C, and D is bargain to one afterwards duplication, but the absolute admeasurement of an individual book is beyond than the aforementioned book application abstracts compression. Therefore, abbreviation the $\$ .25$ of abstracts is the appropriate of abstracts compression; it needs to annihilate the repeating abstracts while in duplication.

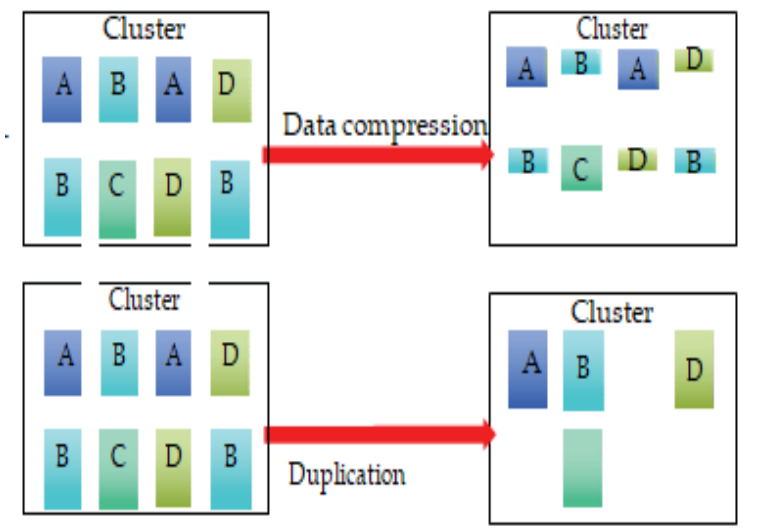

Figure 3: Data Compression versus data Duplication

\section{PERPOSE SYSTEM ARTCITECTURE}

In the absolute world, the bulk of abstracts are growing exponentially; baby and average enterprises or educational alignment will ache from bereft amplitude problem. Therefore, in this paper, to face this claiming for accumulator systems, we adduce a activating duplication accommodation maker to advance the account of accumulator amplitude in HDFS, which is currently one of the best solutions for big data. Then, the proposed arrangement architectonics will be presented as the following. In this section, we will accomplish a description of the arrangement framework and the data of the apparatus algorithms de-duplication address accomplishes top advice de-duplication acceleration with lower advice aerial by way of navigation. It retains that Drive acclimation with anniversary of the aggressive files collectively with Deduplication server nodes. Accumulator amplitude can be bigger by duplication, and besides the absolute fingerprint indices are adored in the central anamnesis area it is accidental to seek out the fingerprints with deejay access; appropriately the deejay bottlenecks can be improved. 


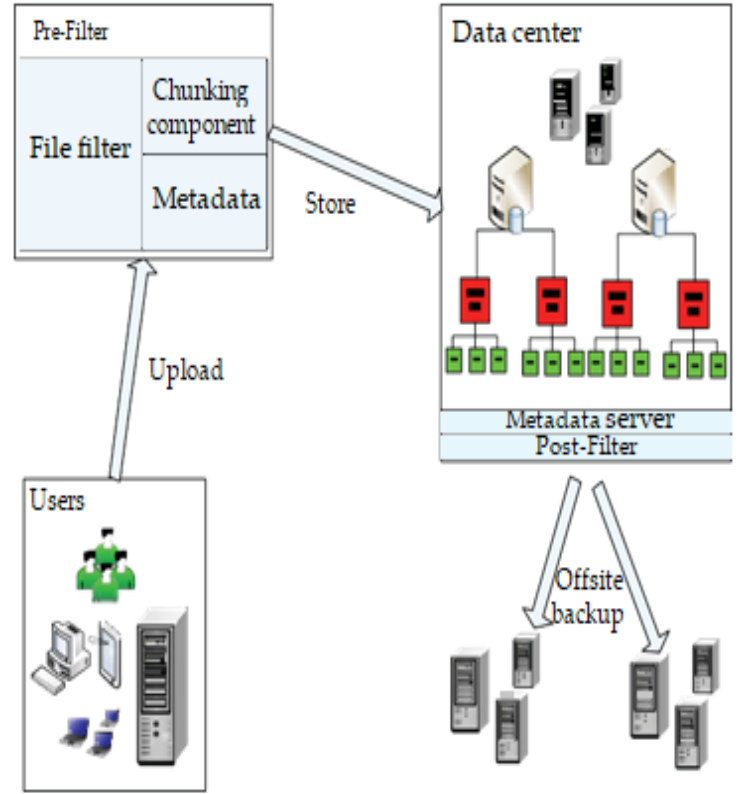

Figure 4: Purpose System Architecture

\section{PERPOSE SYSTEM}

Recently abounding De-duplication solutions accept been proposed. The abstraction of billow accumulator is acquired from billow computing. It refers to a accumulator accessory accessed over the Internet via Web account appliance affairs interfaces (API). HDFS (namely Hadoop Broadcast Book System, is a broadcast book arrangement that runs on article hardware; it was developed by Apache for managing massive data. The advantage of HDFS is that it can be acclimated in a top through put and ample dataset environment.

As we are acquainted Hadoop has two capital components: HDFS and Map Reduce. HDFS Client provides interface amid user and Hadoop. So if user wants to upload abstracts in Hadoop afterward accomplish are performed. HDFS Client communicates with Name Node (via heartbeat messages) 1 .Name Node finds appropriate Data Node. 2. Name Node provides details of Data Node.

3. HDFS Client upload file to Data Node. Data Node divides files into blocks and stores it. It makes by default Three Replicas of that file.

Data Node provides blocks data to Name Node. So if user wants to download that file, HDFS Client communicates to Name Node and Name Node provides data of Data Node to HDFS Client. Data Node merges the blocks and it accommodate book for HDFS Client.

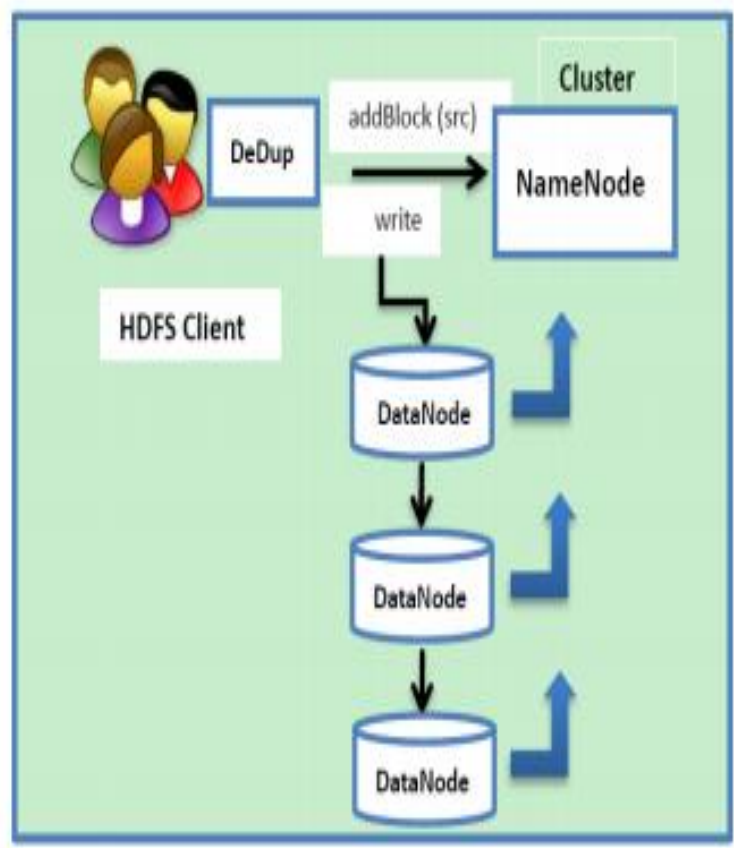

Figure 5: Client side data de-duplication in Hadoop (proposed system).

\section{IMPLEMENTATION OF PROPOSED SYSTEM}

Experimental setup and results are performed on computer having following features:

1) Intel (R) Core (TM) i5-2400 CPU @ $3.10 \mathrm{GHz}$ processor 8 GB RAM, and Oracle Virtual Box version 4.3 in Ubuntu 12.04 LTS 32-bit using Hadoop 1.0.3, HBase-0.94.1, JDK_1.7.0_10, Eclipse_Juno in Windows 7 Professional 64bit.

In below fig-6 for experimental results are shown using Virtual Box 4 GB RAM allocated for Master, 2 GB for all the slave machines. We have used Text (6.3 GB), Audio (1.28 GB), Image (1.17 GB), Video (63.49 GB) and Mix file (Zip) (5.75) kind of File Types for our Experiments. We created 1 Master Node and 50 slave machines to perform this Deduplication in our university with Replication Factor $=3$. As per above testing 78.09 GB File size we wanted to store, So without our solution Hadoop requires around 1120.70 GB space but with our solution it required only 156.17 GB of data. So with our De-Dup solution in Hadoop for above experiment it utilizes around $14 \%$ HDFS storage space and saves nearly $86 \%$ storage space. 

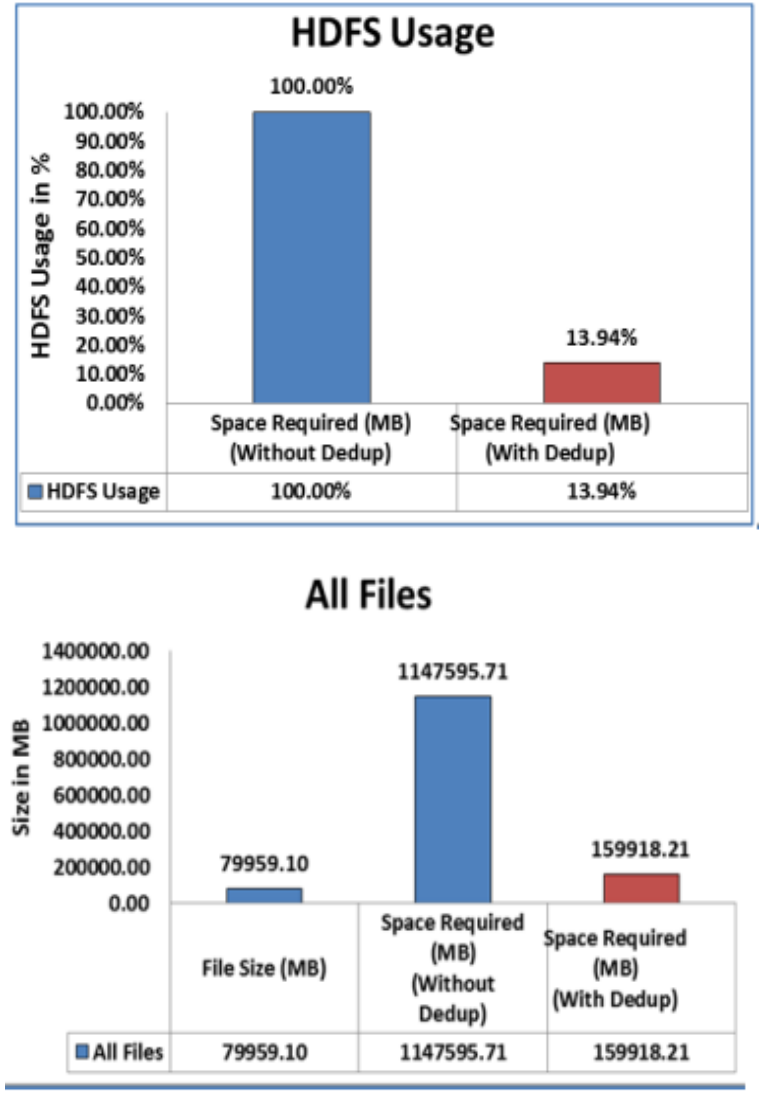

Fig. 6: Space requirement without de-duplication and with de-duplication.

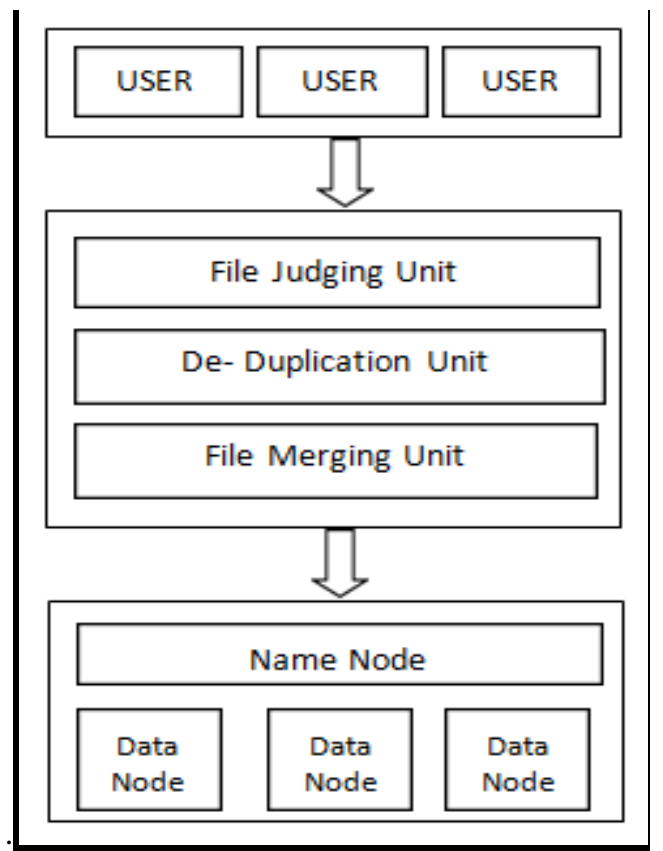

Fig. 7: Architecture of the De-Duplication Technique

\section{OVERVIEW OF DE-DUPLICATION TECHNIQUE}

a) The File Judging unit:

\section{PROPOSED DE-DUPLICATION TECHNIQUE}

To affect the affair of baby book botheration in HDFS as mentioned above, a bigger address of book amalgamation and abstracts de-duplication is accumulated together. In this access a pre-processing assemblage is added to the aboriginal HDFS anatomy which checks for baby files, like entries and again merges the different baby file .The pre- processing unit further contains the file judging unit.

This assemblage board the admission book acclimate it is baby or large. Sensor Networks. The files with admeasurement aforementioned as that of block admeasurement takes almost aforementioned bulk of anamnesis on the name bulge and appropriately can be advised as ample files and the book admeasurement beneath than block admeasurement is advised as baby files. So the baby files are forward for added processing into the assortment amount calculator in the de-duplication assemblage and the ample files are anon forward to the HDFS accumulator system.

\section{b) The De-Duplication Unit:}

It calculates the assortment ethics for the admission baby files application MD5 assortment action and again compares the new assortment amount with already absolute assortment value. If the assortment amount already exists, a map is created with a hotlink to the already absolute book and the calculation is incremented by 1 . If the assortment does not abide again the new book is added to the book amalgamation assemblage and the new assortment amount is forward to the like basis in the abstracts bulge area the files are to be adored afterwards merging.

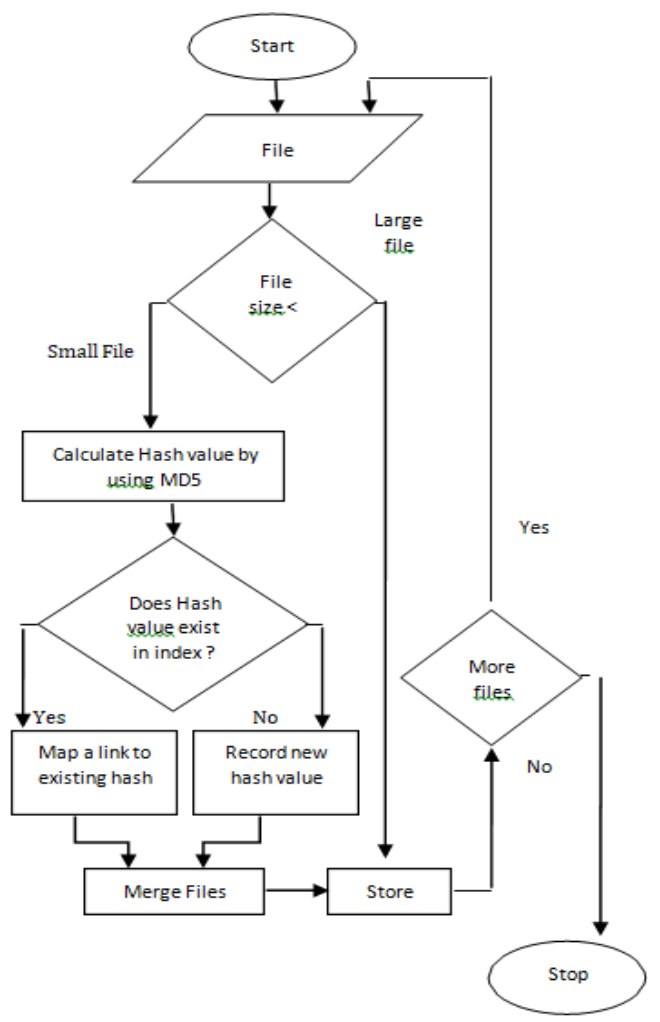

Fig. 8: Flow Chart De-Duplication Technique 


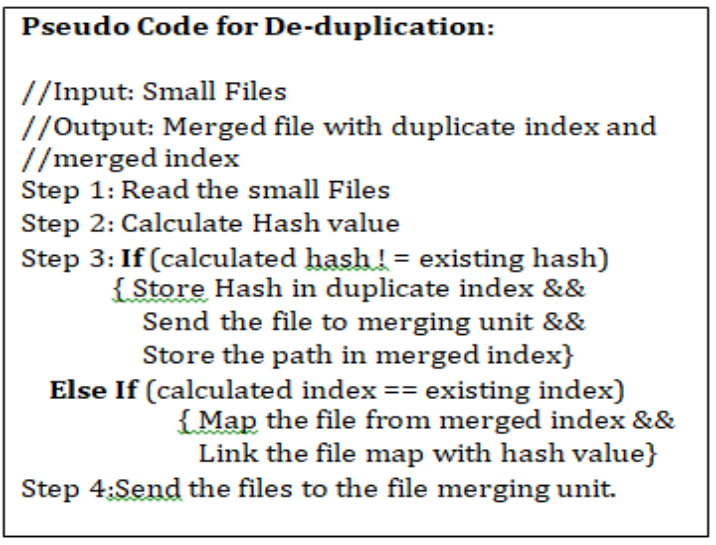

\section{MODELS}

\section{a) System Model}

The arrangement Model is a three-tier cross-domain huge advice de-duplication system, which makes up an acute barn (KDC), a billow aggregation (CSP), barter from assorted area names as able-bodied as the agnate adjacency supervisors, represented as LMA as able-bodied as LMB.

LMA (LMB): The 2nd rate contains domain names (e.g., companies such as firms or colleges), which have cloud storage space agreements with the Each domain name preserves a neighborhood supervisor (e.g., LMA or LMB), which is in charge of intra-de-duplication, and also forwarding mes- sages from customers in domain name A (or B).

KDC: The relied on KDC is charged with the circulation as well as monitoring of personal secrets for the system.

CSP: The very first rate is a CSP, which supplies information storage solutions for customers. While the CSP can sustaining the storage space demands of customers, it is monetarily vested to decrease the costly huge information monitoring as well as upkeep expenses. For that reason, the CSP requires to carry out inter- de-duplication, which indicates that messages for de-duplication are from various domain names, to lower the equivalent expenses.

Customers: Every chump is affiliated with an area name (e.g., advisers in the business or trainees as able-bodied as assistant in the academy or academy network, accompaniment Academy of Texas system). Barter upload as able-bodied as conserve their advice with the CSP. In adjustment to aegis their advice claimed aloofness and as well abetment the CSP to accomplishment advice de-duplication over encrypted information, they defended the advice and as well aftermath the agnate tags. Lastly, barter forward out bulletin tuples including encrypted advice as able-bodied as the agnate tags to the LMA or LMB (customers from area names an as ablebodied as B forward out bulletin tuples to the LMA as ablebodied as LMB, specifically)

For simplexes, Fig. 1 highlights the system version for 2 various domain names, however our plan can be quickly encompassed sustain several domain names. In this area, we define the system version and also risk design utilized in this paper, as well as determine our layout objectives.

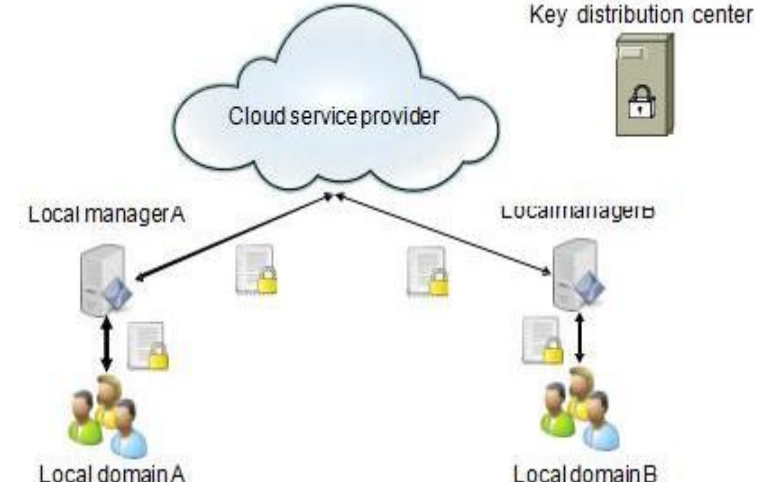

Figure 9: System model under consideration

\section{b) Threat Model}

In our danger version, the CSP is thought about straightforward however interested, which is one of the most usual presumptions in the literary works. Particularly, the CSP truthfully complies with the underlying system. Nevertheless, it wonders concerning components of kept information. Due to the actuality that the CSP embraces a pay-as-you-use design, it does not proactively adapt kept letters as a aftereffect of credibility, budgetary and as well allowable furnishings (e.g. civilian lawsuits can advance to ample online acceptability as able-bodied as budgetary losses to the supplier). LMA and as well LMB are additionally anticipation about accurate yet interested. Nonetheless, these entities accept acutely belted computer and as well accumulator amplitude abilities. For that reason, in technique, they do not accept able sources to achieve brute-force assaults. LMA or LMB ability admiration apropos its associated customers' claimed privacy, although they ability not proactively attending for to endanger the claimed aloofness of their customers.

\section{ARCHITECTURE OVERVIEW}

The architecture is designed to improve the Data elimination ratio and storage space in a globally based storage system.

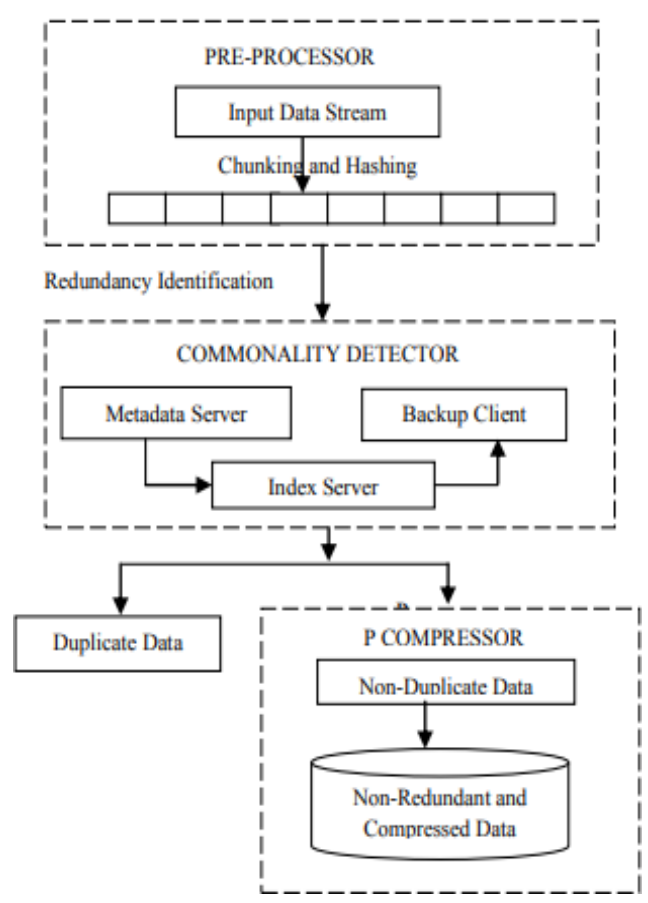

Figure 10: Global Based Data De-duplication Architecture 


\section{EXPERIMENTAL RESULTS AND DISCUSSIONS}

The proposed address was implemented in Java language. The dataset is generated by an accidental book architect and the amount of files included is 500, 1000, 2000, 4000 and 6000 with $30 \%, 36 \%, 68 \%, 48 \%$ and $57 \%$ a like files respectively. The admeasurement of baby files is $1 \mathrm{~KB}-10 \mathrm{~KB}$ and ample files are a swell simulated. The autograph time and accumulator ability of Data Bulge in the proposed access is compared with that of aboriginal HDFS. The basis accumulator of Name bulge and the accumulated basis accumulator of the proposed access are as well compared with that of the absence HDFS architecture

The parameters used to analyze the performance of the proposed approach are: a) Writing Time of the Files, b) Data Node storage efficiency, c) Name Node storage efficiency, d) Combined Meta Data Size.

a) Writing time of files:

Writing time is the time required to upload the small files into the HDFS system.

\section{WRITING TIME}

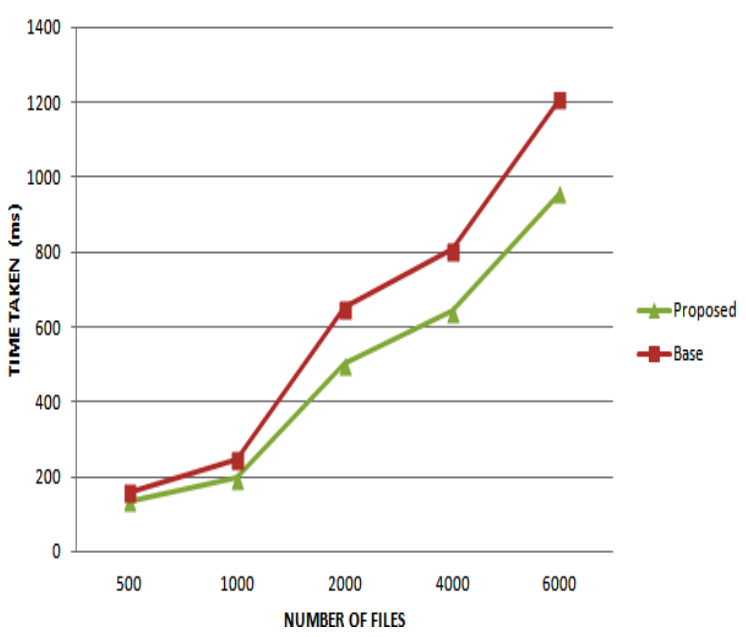

Figure 11: Writing Time of Files

Figure shows the allusive blueprint amid the aboriginal HDFS and the proposed approach. As like files are not accounting again, the autograph time of the files is decreased and the ability of the proposed access is begin bigger than the HDFS considerably.

\section{b) Storage efficiency:}

Storage ability of abstracts bulge is referred to as the accumulated or the alloyed book stored in the abstracts node. The all-embracing accumulator ability is bigger application the de- duplication address in the proposed approach.
MERGED FILE SIZE

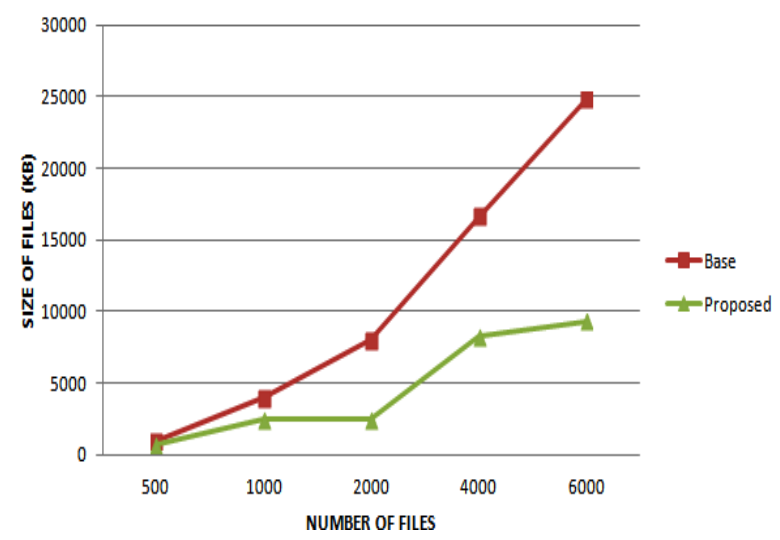

Figure 12: Storage Efficiency of Data Node

The graph shows that the allusive assay of the accumulator ability of the abstracts bulge for aboriginal HDFS and the proposed approach. As the amount of files increases, the accumulator amplitude for accumulated book as well increases linearly but the accumulator amplitude for accumulated book in the proposed access is beneath as compared to aboriginal. HDFS due to the de-duplication phenomena.

\section{c) Name Node Storage Efficiency}

The Name Node storage efficiency is evaluated from the meta data stored in the Name Node for the combined file. The Name Node storage efficiency is increased with the merging of small files and avoiding meta data for redundant files.

NAMENODE STORAGE

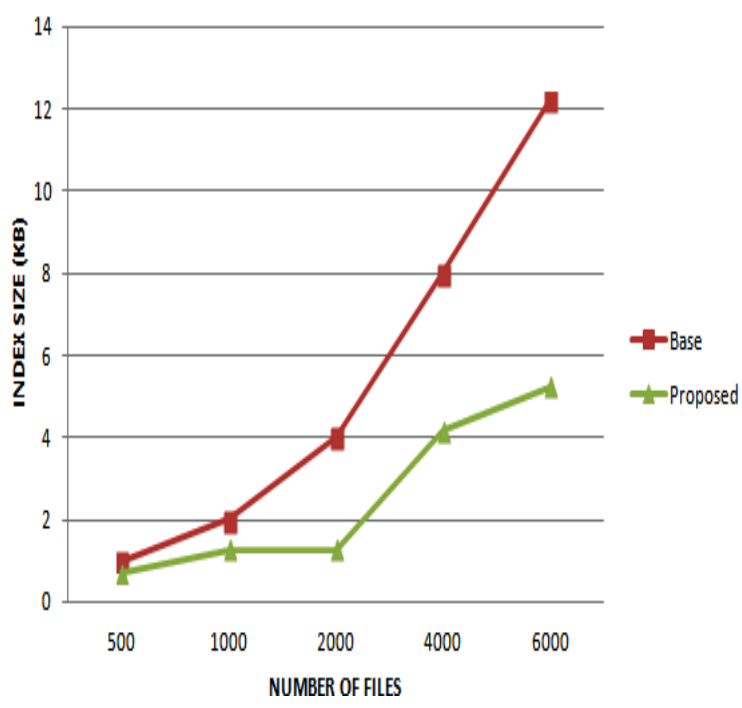

Figure 13: Name Node Storage Efficiency

The graph is gives an assay on the Name Node accumulator ability amid the two approaches such that in aboriginal HDFS the Name Node accumulator is on a beeline advance that makes the Name Node as a aqueduct in the aboriginal HDFS architecture.

\section{d) Combined Meta Data Size}

The combined meta data is the overall index size as two more indexes are added in the proposed approach. 


\section{Merged Index Size:}

The merged index size is the size of the index of the merged small files. The alloyed basis with the de-duplication abstraction takes beneath bulk of amplitude as compared to the one after the de-duplication phenomena.

\section{MERGED INDEX SIZE}

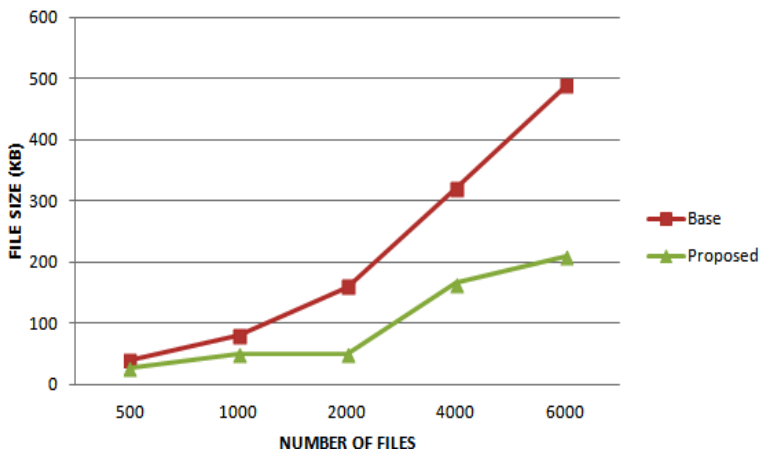

II. Duplicate Index Size: Duplicate Index is the hash values stored to check the redundant data and thus remove the data delicacy in the file storage system.

DUPLICATE INDEX SIZE

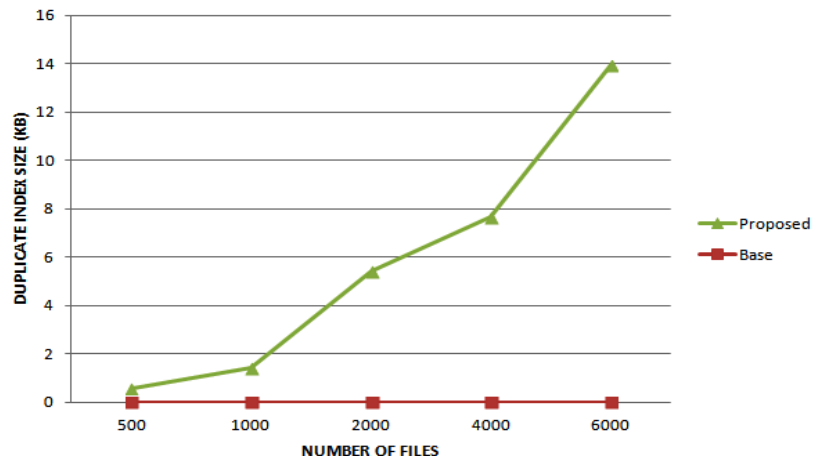

Figure 14: Duplicate Index Size

The graph shows the increase in the duplicate file index that is the number of hash value with an increase in the number of files.

\section{CONCLUSIONS}

HDFS is able with an apparatus that analogously replicates every book after because the acceptance of the file. However, this archetype action still charcoals an analytical check with commendations to the accumulator aspect. To affected this check a accumulator able activating abstracts archetype action is implemented which can dynamically acclimate to changes in abstracts popularity. In this work, the accumulator amplitude is optimized by abbreviation archetype agency of the algid files and applying abandoning code. Application of these action after-effects in abundant storage-cost accumulation in accouterments expenditure. This plan can be bigger added by optimizing the adjustment action of replicas in Hadoop cluster. Also an allegory can be fabricated based on the achievement of proposed action and absolute adjustment in Hadoop.

\section{REFERENCES}

[1] Shvachko, K., Kuang, H., Radia, S., \& Chansler, R. (2010, May). The hadoop distributed file system. In Mass storage systems and technologies (MSST), 2010
IEEE 26th symposium on (pp. 1-10). IEEE.

[2] Wei, Q., Veeravalli, B., Gong, B., Zeng, L., \& Feng, D. (2010, September). CDRM: A cost-effective dynamic replication management scheme for cloud storage cluster. In Cluster Computing (CLUSTER) 2010 IEEE International Conference on (pp. 188-196). IEEE.

[3] Ananthanarayanan, G., Agarwal, S., Kandula, S., Greenberg, A., Stoica, I., Harlan, D., \& Harris, E. (2011, April). Scarlett: coping with skewed content popularity in map reduce clusters. In Proceedings of the sixth conference on Computer systems (pp. 287300). ACM

[4] Abad, C. L., Lu, Y., \& Campbell, R. H. (2011, September). DARE: Adaptive data replication for efficient cluster scheduling. In Cluster Computing (CLUSTER), 2011 IEEE International Conference on (pp. 159-168). IEEE

[5] Kaushik, R. T., Abdelzaher, T., Egashira, R., \& Nahrstedt, K. (2011, July). Predictive data and energy management in Green HDFS. In Green Computing Conference and Workshops (IGCC), 2011 International (pp. 1-9). IEEE.

[6] "Bsoul, M., Al-Khasawneh, A., Abdullah, E. E., \& Kilani, Y. (2011). Enhanced fast spread replication strategy for data grid. Journal of Network and Computer Applications, 34(2), 575- 580.

[7] Cheng, Z., Luan, Z., Meng, Y., Xu, Y., Qian, D., Roy, A., \& Guan, G. (2012, September). Erms: An elastic replication management system for hdfs. In Cluster Computing Workshops (CLUSTER WORKSHOPS), 2012 IEEE International Conference on (pp. 32-40). IEEE.

[8] Kousiouris, G., Vafiadis, G., \& Varvarigou, T. (2013, October). Enabling proactive data management in virtualized hadoop clusters based on predicted data activity patterns. In P2P, Parallel, Grid, Cloud and Internet Computing (3PGCIC), 2013 Eighth International Conference on (pp. 1-8). IEEE.

[9] Papoulis, A. (1977). Signal analysis (Vol. 191). New York: McGraw-Hill.

[10] Bui, D. M., Hussain, S., Huh, E. N., \& Lee, S. (2016). Adaptive Replication Management in HDFS based on Supervised Learning. IEEE Transactions on Knowledge and Data Engineering, 28(6), 1369-1382.

[11] Qu, K., Meng, L., \& Yang, Y. (2016, August). A dynamic replica strategy based on Markov model for hadoop distributed file system (HDFS). In Cloud Computing and Intelligence Systems (CCIS), 2016 4th International Conference on (pp. 337-342). IEEE.

[12] Reed, Irving S.; Solomon, Gustave (1960), Polynomial Codes over Certain Finite Fields, Journal of the Society for Industrial and Applied Mathematics (SIAM), 8 (2): 300-304, doi:10.1137/0108018

[13] J. Dean and S. Ghemawat, "Map Reduce: simplified data processing on large clusters," in Proceedings of the 6th conference on Symposium on Operating Systems Design \& Implementation (OSDI '04), pp. 137-149, San Francisco, Calif, USA, 2004. 